\title{
Large almost monochromatic subsets in hypergraphs
}

\author{
David Conlon* Jacob Fox ${ }^{\dagger} \quad$ Benny Sudakov ${ }^{\ddagger}$
}

\begin{abstract}
We show that for all $\ell$ and $\epsilon>0$ there is a constant $c=c(\ell, \epsilon)>0$ such that every $\ell$-coloring of the triples of an $N$-element set contains a subset $S$ of size $c \sqrt{\log N}$ such that at least $1-\epsilon$ fraction of the triples of $S$ have the same color. This result is tight up to the constant $c$ and answers an open question of Erdős and Hajnal from 1989 on discrepancy in hypergraphs. For $\ell \geq 4$ colors, it is known that there is an $\ell$-coloring of the triples of an $N$-element set whose largest monochromatic subset has cardinality only $\Theta(\log \log N)$. Thus, our result demonstrates that the maximum almost monochromatic subset that an $\ell$-coloring of the triples must contain is much larger than the corresponding monochromatic subset. This is in striking contrast with graphs, where these two quantities have the same order of magnitude. To prove our result, we obtain a new upper bound on the $\ell$-color Ramsey numbers of complete multipartite 3-uniform hypergraphs, which answers another open question of Erdős and Hajnal.
\end{abstract}

\section{Introduction}

The Ramsey number $r(n)$ is the smallest integer $N$ such that every 2-coloring of the edges of the complete graph on $N$ vertices contains a monochromatic clique of size $n$. Ramsey's theorem states that $r(n)$ exists for all $n$. Determining or estimating Ramsey numbers is one of the central problems in combinatorics, see the book Ramsey theory [12] for details. A classical result of Erdős and Szekeres [11, which is a quantitative version of Ramsey's theorem, implies that $r(n) \leq 2^{2 n}$ for every positive integer $n$. Erdős [5] showed using probabilistic arguments that $r(n)>2^{n / 2}$ for $n>2$. Over the last sixty years, there have been several improvements on these bounds (see, e.g., [3]). However, despite efforts by various researchers, the constant factors in the above exponents remain the same.

Although already for graph Ramsey numbers there are significant gaps between lower and upper bounds, our knowledge of hypergraph Ramsey numbers is even weaker. The Ramsey number $r_{k}(n)$ is the minimum $N$ such that every 2-coloring of the $k$-tuples of an $N$-element set contains a monochromatic set of size $n$, where a set is called monochromatic if all its $k$-tuples have the same color. Erdös, Hajnal, and Rado [9] showed that there are positive constants $c$ and $c^{\prime}$ such that

$$
2^{c n^{2}}<r_{3}(n)<2^{2^{c^{\prime} n}}
$$

\footnotetext{
${ }^{*}$ St John's College, Cambridge, United Kingdom. E-mail: D.Conlon@dpmms.cam.ac.uk. Research supported by a research fellowship at St John's College.

${ }^{\dagger}$ Department of Mathematics, Princeton, Princeton, NJ. Email: jacobfox@math.princeton.edu. Research supported by an NSF Graduate Research Fellowship and a Princeton Centennial Fellowship.

${ }^{\ddagger}$ Department of Mathematics, UCLA, Los Angeles, CA 90095. Email: bsudakov@math.ucla.edu. Research supported in part by NSF CAREER award DMS-0812005 and by USA-Israeli BSF grant.
} 
They also conjectured that $r_{3}(n)>2^{2^{c n}}$ for some constant $c>0$ and Erdös (see, e.g. [2]) offered a $\$ 500$ reward for a proof. Similarly, for $k \geq 4$, there is a difference of one exponential between known upper and lower bounds for $r_{k}(n)$, i.e.,

$$
t_{k-1}\left(c n^{2}\right) \leq r_{k}(n) \leq t_{k}\left(c^{\prime} n\right),
$$

where the tower function $t_{k}(x)$ is defined by $t_{1}(x)=x$ and $t_{i+1}(x)=2^{t_{i}(x)}$.

The study of 3-uniform hypergraphs is particularly important for our understanding of hypergraph Ramsey numbers. This is because of an ingenious construction called the stepping-up lemma due to Erdős and Hajnal (see, e.g., Chapter 4.7 in [12]). For $k \geq 3$, their method allows one to construct lower bound colorings for uniformity $k+1$ from colorings for uniformity $k$, effectively gaining an extra exponential each time it is applied. Therefore, proving that $r_{3}(n)$ has doubly exponential growth will allow one to close the gap between the upper and lower bounds for $r_{k}(n)$ for all uniformities $k$.

Despite the fact that Erdős [6] (see also the book [2]) believed $r_{3}(n)$ is closer to $2^{2^{c n}}$, together with Hajnal [8], he discovered the following interesting fact about hypergraphs which maybe indicates the opposite. They proved that there are $c, \epsilon>0$ such that every 2-coloring of the triples of an $N$-element set contains a subset $S$ of size $s>c(\log N)^{1 / 2}$ such that at least $(1 / 2+\epsilon)\left(\begin{array}{l}s \\ 3\end{array}\right)$ triples of $S$ have the same color. That is, this subset deviates from having density $1 / 2$ in each color by at least some fixed positive constant. Erdös [7] further remarks that he would begin to doubt that $r_{3}(n)$ is double-exponential in $n$ if one can prove that any 2-coloring of the triples of an $N$-set contains some set of size $s=c(\epsilon)(\log N)^{\delta}$ for which at least $(1-\epsilon)\left(\begin{array}{l}s \\ 3\end{array}\right)$ triples have the same color, where $\delta>0$ is an absolute constant. Erdos and Hajnal proposed [8] that such a statement may even be true with $\delta=1 / 2$. Our first result shows that this is indeed the case.

Theorem 1 For each $\epsilon>0$ and $\ell$, there is $c=c(\ell, \epsilon)>0$ such that every $\ell$-coloring of the triples of an $N$-element set contains a subset $S$ of size $s=c \sqrt{\log N}$ such that at least $(1-\epsilon)\left(\begin{array}{l}s \\ 3\end{array}\right)$ triples of $S$ have the same color.

It is easy to see that this theorem is tight up to the constant factor $c$. Indeed, consider an $\ell$-coloring of the triples of an $N$-element set in which every triple gets one of $\ell$ colors uniformly at random. Using a standard tail estimate for the binomial distribution, one can show that in this coloring, with high probability, every subset of size $\gg \sqrt{\log N}$ has a $1 / \ell+o(1)$ fraction of its triples in each color.

Our result also shows a significant difference between the discrepancy problem in graphs and that in hypergraphs. The $\ell$-color Ramsey number $r_{k}(n ; \ell)$ is the minimum $N$ such that every $\ell$-coloring of the $k$-tuples of an $N$-element set contains a monochromatic set of size $n$. Erdős and Hajnal (see, e.g., [12]) constructed a 4-coloring of the triples of a set of size $2^{2^{c n}}$ which does not contain a monochromatic subset of size $n$. In [4] we constructed a 3-coloring of the triples of a set of size $2^{n^{c \log n}}$ which does not contain a monochromatic subset of size $n$. Thus, Theorem 1 demonstrates (at least for $\ell \geq 3$ ) that the maximum almost monochromatic subset that an $\ell$-coloring of the triples must contain is much larger than the corresponding monochromatic subset. This is in striking contrast with graphs, where these two quantities have the same order of magnitude, as demonstrated by a random $\ell$-coloring of the edges of a complete graph.

Another open problem from the 1989 paper of Erdös and Hajnal [8] asks whether one can exhibit a fixed hypergraph of density larger than $1 / 2+\epsilon$ on $c \sqrt{\log N}$ vertices that occurs monochromatically. 
That is, can we find dense hypergraphs with small Ramsey numbers? We show that this is indeed the case by obtaining a new upper bound on the $\ell$-color Ramsey number of a complete multipartite 3-uniform hypergraph. A hypergraph $H=(V, E)$ consists of a vertex set $V$ and an edge set $E$, which is a collection of subsets of $V$. A hypergraph is $k$-uniform if each edge has exactly $k$ vertices. For a $k$-uniform hypergraph $H$, the Ramsey number $r(H ; \ell)$ is the minimum $N$ such that every $\ell$-coloring of the $k$-tuples of an $N$-element set contains a monochromatic copy of $H$. The complete d-partite $k$-uniform hypergraph $K_{d}^{k}(n)$ is the $k$-uniform hypergraph whose vertex set consists of $d$ parts of size $n$ and whose edges are all $k$-tuples that have their vertices in some $k$ different parts. The number of vertices of $K_{d}^{3}(n)$ is $d n$ and the number of edges in $K_{d}^{3}(n)$ is $\left(\begin{array}{l}d \\ 3\end{array}\right) n^{3}>\left(1-\frac{3}{d}\right)\left(\begin{array}{c}d n \\ 3\end{array}\right)$, i.e., it has edge density more than $1-\frac{3}{d}$. In particular, as $d$ increases, the edge density of $K_{d}^{3}(n)$ tends to 1 . Therefore, Theorem 1 is an immediate corollary of the following theorem.

Theorem 2 The $\ell$-color Ramsey number of the complete d-partite hypergraph $K_{d}^{3}(n)$ satisfies

$$
r\left(K_{d}^{3}(n) ; \ell\right) \leq 2^{\ell^{2 r} n^{2}},
$$

where $r=r_{2}(d-1 ; \ell)$ is the $\ell$-color Ramsey number of the complete graph on $d-1$ vertices.

In the next section, we discuss the technique of Kövári, Sós, and Turán on the classical problem of Zarankiewicz and its consequences, which will be helpful in proving our main theorems. In Section 3 , we present the proof of Theorem 2, Throughout the paper, we systematically omit floor and ceiling signs whenever they are not crucial for the sake of clarity of presentation. We also do not make any serious attempt to optimize absolute constants in our statements and proofs. All logarithms in this paper are in base 2 .

\section{Complete bipartite graphs in dense graphs}

The problem of Zarankiewicz [15] asks for the maximum number $z(m, n ; s, t)$ of edges in a bipartite graph $G$ which has $m$ vertices in its first class, $n$ vertices in its second class, and does not contain a complete bipartite graph $K_{s, t}$ with $s$ vertices in the first class and $t$ in the other. In their celebrated paper, Kövári, Sós, and Turán [13] used double counting together with the pigeonhole principle to give a general upper bound on $z(m, n ; s, t)$. Using this technique, we obtain the following two simple lemmas which we need in the proof of our main result. The degree $d(v)$ of a vertex $v$ is the number of vertices adjacent to $v$.

Lemma 1 Let $G$ be a bipartite graph with parts $A$ and $B$ and with at least $|A||B| / \ell$ edges. Then $G$ contains a complete bipartite subgraph with one part having $a=|A| / \ell$ vertices from $A$ and the other part having $b=2^{-|A|}|B|$ vertices from $B$.

Proof: Using the convexity of the function $f(x)=\left(\begin{array}{l}x \\ a\end{array}\right)$ together with the fact that the average degree of a vertex in $B$ is at least $a$, we conclude that the number of pairs $\left(A^{\prime}, v\right)$, where $A^{\prime}$ is a subset of $A$ of size $a$ and $v \in B$ is adjacent to every vertex in $A^{\prime}$, is at least

$$
\sum_{v \in B}\left(\begin{array}{c}
d(v) \\
a
\end{array}\right) \geq|B|\left(\begin{array}{c}
\frac{1}{|B|} \sum_{v \in B} d(v) \\
a
\end{array}\right)=|B| .
$$


Since the set $A$ has at most $2^{|A|}$ subsets, by the pigeonhole principle, there are at least $b=2^{-|A|}|B|$ vertices in $B$ adjacent to the same subset $A^{\prime} \subset A$ of size $a$. Together they form a complete bipartite graph with one part having $a$ vertices in $A$ and the other part having $b$ vertices in $B$, completing the proof.

Lemma 2 If a graph $G$ of order $n$ has $\epsilon n^{2}$ edges and $t<\epsilon n$, then it contains $K_{s, t}$ with $s=\epsilon^{t} n$.

Proof: Note that the number of pairs $(U, v)$ with $U$ being a subset of $G$ of size $t$ and $v$ being a vertex of $G$ adjacent to every vertex in $U$ is at least

$$
\sum_{v \in V(G)}\left(\begin{array}{c}
d(v) \\
t
\end{array}\right) \geq n\left(\begin{array}{c}
2 \epsilon n \\
t
\end{array}\right) \geq n(\epsilon n)^{t} / t !
$$

where we use convexity of $f(x)=\left(\begin{array}{l}x \\ t\end{array}\right)$ and the fact that the average degree of a vertex in $G$ is $2 \epsilon n>t+\epsilon n$. If $G$ does not contain $K_{s, t}$ as a subgraph, then for every subset $U$ of $G$ of size $t$, there are at most $s-1$ vertices of $G$ adjacent to $U$. Hence,

$$
n(\epsilon n)^{t} / t ! \leq(s-1)\left(\begin{array}{l}
n \\
t
\end{array}\right)<s n^{t} / t !=n(\epsilon n)^{t} / t !
$$

a contradiction, which completes the proof.

Both of these lemmas can be further improved by using better upper bound estimates for binomial coefficients. However, the above clean estimates are sufficient for our purposes.

\section{Proof of the main result}

First we briefly discuss the classical approach of Erdős and Rado which gives an upper bound on the Ramsey number of the complete 3-uniform hypergraph on $d$ vertices. Suppose the triples of a sufficiently large set $V$ of vertices are $\ell$-colored. Let $r$ be the $\ell$-color Ramsey number of a complete graph of order $d-1$. Erdős and Rado greedily construct a set of vertices $\left\{v_{1}, \ldots, v_{r+1}\right\}$ such that for any given pair $1 \leq i<j \leq r$, all triples $\left\{v_{i}, v_{j}, v_{k}\right\}$ with $k>j$ are of the same color, which we denote by $\chi\left(v_{i}, v_{j}\right)$. By definition of the Ramsey number $r=r_{2}(d-1 ; \ell)$, there is a monochromatic clique of size $d-1$ in coloring $\chi$, and this clique together with $v_{r+1}$ forms a monochromatic set of size $d$ in the original coloring. Note that in their approach, after having picked $\left\{v_{1}, \ldots, v_{i}\right\}$, we have a subset $S_{i}$ such that for any pair $a, b$ with $1 \leq a<b \leq i$, all triples $\left\{v_{a}, v_{b}, w\right\}$ with $w \in S_{i}$ are the same color. The subset $S_{i}$ consists of those vertices from which we can draw future vertices.

Instead of picking vertices one by one as in the Erdős-Rado technique, we instead pick subsets one by one. After step $i$, we will have disjoint subsets $V_{1, i}, \ldots, V_{i, i}$ and another subset $S_{i}$ such that for any pair $a, b$ with $1 \leq a<b \leq i$, all triples in $V_{a, i} \times V_{b, i} \times V_{c, i}$ with $b<c \leq i$ and all triples in $V_{a, i} \times V_{b, i} \times S_{i}$ are the same color. Similar to before, the subset $S_{i}$ consists of those vertices from which we can draw future vertex subsets. 
We now present the proof of Theorem 2, which states that the $\ell$-color Ramsey number of the complete $d$-partite hypergraph $K_{d}^{3}(n)$ satisfies

$$
r\left(K_{d}^{3}(n) ; \ell\right) \leq 2^{\ell^{2 r} n^{2}}
$$

where $r=r_{2}(d-1 ; \ell)$. The set of edges of a graph $G$ is denoted by $E(G)$ and $e(G)=|E(G)|$.

Proof of Theorem 2: Let $r=r_{2}(d-1 ; \ell)$ and let $V$ be a set of $N=2^{\ell^{2 r} n^{2}}$ vertices whose triples are $\ell$-colored. We will construct disjoint subsets $V_{1}, V_{2}, \ldots, V_{r+1}$ of size $n$ such that for each pair $1 \leq i<j \leq r$ there is a color $\chi(i, j)$ for which all triples in $V_{i} \times V_{j} \times V_{k}$ with $j<k \leq r+1$ have color $\chi(i, j)$. Note that $\chi$ is an $\ell$-coloring of the edges of the complete graph with vertex set $\{1, \ldots, r\}$ and therefore, from the definition of the Ramsey number $r=r_{2}(d-1 ; \ell)$, it follows that there are $d-1$ numbers $1 \leq i_{1}<i_{2}<\ldots<i_{d-1} \leq r$ that make a monochromatic clique in coloring $\chi$. Then, using the properties of $\chi$ it is easy to see that the corresponding sets $V_{i_{1}}, V_{i_{2}}, \ldots, V_{i_{d-1}}$ together with $V_{r+1}$ make a monochromatic $K_{d}^{3}(n)$. So we are left with constructing subsets $V_{1}, \ldots, V_{r+1}$ with the desired properties, which we will do in $r$ rounds.

In the first round we pick $V_{1,1} \subset V$ of size $\ell^{-1} \sqrt{\log N}$ arbitrarily and let $S_{1}=V \backslash V_{1,1}$, so $\left|S_{1}\right|=$ $N-\ell^{-1} \sqrt{\log N} \geq N^{3 / 4}$. Now suppose that we already constructed disjoint subsets $V_{1, i}, V_{2, i}, \ldots, V_{i, i}$ each of size $\ell^{-i} \sqrt{\log N}$ and a subset $S_{i}$ of vertices disjoint from $V_{1, i}, \ldots, V_{i, i}$ with $\left|S_{i}\right| \geq N^{1 / 4+2^{-i}}$ such that if $1 \leq a<b \leq i$, then all triples in $V_{a, i} \times V_{b, i} \times V_{c, i}$ with $b<c \leq i$ and all triples in $V_{a, i} \times V_{b, i} \times S_{i}$ have color $\chi(a, b)$. Note that this is satisfied for $i=1$.

We next show how we proceed through round $i+1$. Consider all triples with one vertex in $V_{1, i}$ and the other two vertices in $S_{i}$. By the pigeonhole principle, at least a $1 / \ell$ fraction of these triples have the same color which we denote by $\chi(1, i+1)$. Let $H$ be an auxiliary bipartite graph whose first part $A$ is $V_{1, i}$, second part $B$ consists of all the unordered pairs $\left(w, w^{\prime}\right)$ from $S_{i}$, and whose edges are those pairs $(a, b), a \in A$ and $b=\left(w, w^{\prime}\right) \in B$ such that the triple $\left(a, w, w^{\prime}\right)$ has color $\chi(1, i+1)$. By applying Lemma1 to $H$ we find a subset $V_{1, i+1} \subset V_{1, i}$ with $\left|V_{1, i+1}\right|=\left|V_{1, i}\right| / \ell=\ell^{-(i+1)} \sqrt{\log N}$ and a graph $G_{1, i}$ on $S_{i}$ with at least

$$
2^{-\left|V_{1, i}\right|}\left(\begin{array}{c}
\left|S_{i}\right| \\
2
\end{array}\right) \geq 2^{-2-\ell^{-i} \sqrt{\log N}}\left|S_{i}\right|^{2}
$$

edges such that all triples consisting of a vertex from $V_{1, i+1}$ and an edge from $G_{1, i}$ have color $\chi(1, i+1)$.

Continuing this process, suppose that after $j$ steps, we have already picked for all $1 \leq h \leq j$ the sets $V_{h, i+1} \subset V_{h, i}$ with $\left|V_{h, i+1}\right|=\left|V_{h, i}\right| / \ell=\ell^{-(i+1)} \sqrt{\log N}$ and graphs $G_{j, i} \subset G_{j-1, i} \subset \ldots \subset G_{1, i}$ on $S_{i}$ which have the following properties. The number of edges of $G_{j, i}$ is at least

$$
2^{-2-j \ell^{-i} \sqrt{\log N}}\left|S_{i}\right|^{2}
$$

and every triple consisting of a vertex in $V_{h, i+1}$ together with the two vertices of an edge from $G_{h, i}$ have color $\chi(h, i+1)$.

In the step $j+1$, consider all triples whose one vertex is in $V_{j+1, i}$ and the other two vertices form an edge of $G_{j, i}$. By the pigeonhole principle, at least a $1 / \ell$ fraction of these triples have the same color, which we denote by $\chi(j+1, i+1)$. Similar to before, let $H$ be an auxiliary bipartite graph whose first part $A$ is $V_{j+1, i}$, second part $B$ consists of all the edges of $G_{j, i}$, and the edges of $H$ are those pairs $(a, b), a \in A$ and $b=\left(w, w^{\prime}\right) \in E\left(G_{j, i}\right)$ such that the triple $\left(a, w, w^{\prime}\right)$ has color $\chi(j+1, i+1)$. By 
applying Lemma1 1 to $H$ we find a subset $V_{j+1, i+1} \subset V_{j+1, i}$ with $\left|V_{j+1, i+1}\right|=\left|V_{j+1, i}\right| / \ell=\ell^{-(i+1)} \sqrt{\log N}$ and a subgraph $G_{j+1, i} \subset G_{j, i} \subset \ldots \subset G_{1, i}$ on $S_{i}$ with at least

$$
2^{-\left|V_{j+1, i}\right|} e\left(G_{j, i}\right) \geq 2^{-\left|V_{j+1, i}\right|} 2^{-2-j \ell^{-i} \sqrt{\log N}}\left|S_{i}\right|^{2}=2^{-2-(j+1) \ell^{-i} \sqrt{\log N}}\left|S_{i}\right|^{2}
$$

edges such that all triples whose one vertex is from $V_{j+1, i+1}$ and the other two vertices form an edge from $G_{j+1, i}$ have color $\chi(j+1, i+1)$.

After $i$ such steps, we have $V_{1, i+1}, \ldots, V_{i, i+1}$ each of size $\ell^{-(i+1)} \sqrt{\log N}$ and a sequence of graphs $G_{i, i} \subset G_{i-1, i} \subset \ldots \subset G_{1, i}$ on $S_{i}$ such that the number of edges of $G_{i, i}$ is at least

$$
2^{-2-i \ell^{-i} \sqrt{\log N}}\left|S_{i}\right|^{2} \geq 2^{-\sqrt{\log N}}\left|S_{i}\right|^{2}
$$

and, for $1 \leq h \leq i$, all triples consisting of a vertex in $V_{h, i+1}$ together with an edge from $G_{h, i}$ are color $\chi(h, i+1)$.

Now we apply Lemma 2 (with $\epsilon=2^{-\sqrt{\log N}}$ and $t=\ell^{-(i+1)} \sqrt{\log N}$ ) to graph $G_{i, i}$ to find disjoint subsets $V_{i+1, i+1}$ and $S_{i+1}$ of $S_{i}$ that form a complete bipartite graph in $G_{i, i}$ and satisfy $\left|V_{i+1, i+1}\right|=$ $\ell^{-(i+1)} \sqrt{\log N}$, and

$$
\left|S_{i+1}\right| \geq\left(2^{-\sqrt{\log N}}\right)^{\left|V_{i+1, i+1}\right|}\left|S_{i}\right|=N^{-\ell^{-(i+1)}}\left|S_{i}\right| \geq N^{-2^{-(i+1)}} N^{1 / 4+2^{-i}} \geq N^{1 / 4+2^{-(i+1)}} .
$$

By construction we have that for $j<i+1$ all triples in $V_{j, i+1} \times V_{i+1, i+1} \times S_{i+1}$ have color $\chi(j, i+1)$. On the other hand, since $V_{j, i+1} \subset V_{j, i}$ for $j<i+1$ and $V_{i+1, i+1}, S_{i+1} \subset S_{i}$ we have by induction that if $1 \leq a<b \leq i$, then all triples in $V_{a, i+1} \times V_{b, i+1} \times V_{c, i+1}$ with $b<c \leq i+1$ and all triples in $V_{a, i+1} \times V_{b, i+1} \times S_{i+1}$ have color $\chi(a, b)$. This completes the description of round $i+1$ of our induction process.

After $r$ such iterations, we have disjoint subsets $V_{1, r}, \ldots, V_{r, r}$, each of size $\ell^{-r} \sqrt{\log N}=n$ and $S_{r}$ of size at least $N^{1 / 4} \geq n$ such that if $1 \leq a<b \leq r$, then all triples in $V_{a, r} \times V_{b, r} \times V_{c, r}$ with $b<c \leq r$ and all triples in $V_{a, r} \times V_{b, r} \times S_{r}$ have color $\chi(a, b)$. Letting $V_{i}=V_{i, r}$ for $1 \leq i \leq r$ and $V_{r+1}$ to be any subset of $S_{r}$ of size $n$, we obtain the sets $V_{1}, \ldots, V_{r+1}$ with desired properties. This completes the proof.

\section{Concluding remarks}

- It would be very interesting to extend Theorems 1 and 2 to uniformity $k \geq 4$. In [4] we obtain some preliminary remarks in this direction. We show that for all $k, \ell$ and $\epsilon>0$ there is $\delta=\delta(k, \ell, \epsilon)>0$ such that every $\ell$-coloring of the $k$-tuples of an $N$-element set contains a subset of size $s=(\log N)^{\delta}$ which contains at least $(1-\epsilon)\left(\begin{array}{l}s \\ k\end{array}\right) k$-tuples of the same color. Unfortunately, notice that $\delta$ here depends on $\epsilon$. Just as we deduce Theorem 1 from Theorem 2 , this result is obtained by proving an upper bound on the Ramsey numbers of complete multipartite $k$-uniform hypergraphs.

- It would be nice to determine the best possible dependence of $c$ on $\epsilon$ in Theorem 1, As we already mention in the introduction, this theorem follows from our bound on the $\ell$-color Ramsey number 
of the complete $d$-partite 3-uniform hypergraph with $d=\Theta\left(\epsilon^{-1}\right)$. From Theorem 2 we have that $c \leq \ell^{-r}$, where $r$ is the $\ell$-color Ramsey number of a complete graph of order $d-1$. Therefore,

using the simple upper bound $r=r_{2}(d-1 ; \ell) \leq \ell^{(d-1) \ell}$ we obtain that $c(\ell, \epsilon) \leq 2^{-\ell^{\Theta(\ell / \epsilon)}}$. It seems likely that this double exponential dependence on $1 / \epsilon$ is not correct.

\section{References}

[1] B. Bollobás, Extremal Graph Theory, Dover Publications, Inc., Mineola, NY, 2004. Reprint of the 1978 Original.

[2] F. Chung and R. Graham, Erdős on Graphs. His Legacy of Unsolved Problems, A K Peters, Ltd., Wellesley, MA, 1998.

[3] D. Conlon, A new upper bound for diagonal Ramsey numbers, Annals of Mathematics, to appear.

[4] D. Conlon, J. Fox, and B. Sudakov, Hypergraph Ramsey numbers, submitted.

[5] P. Erdős, Some remarks on the theory of graphs, Bull. Amer. Math. Soc. 53 (1947), 292-294.

[6] P. Erdös, Problems and results on graphs and hypergraphs: similarities and differences, in Mathematics of Ramsey theory, Algorithms Combin., Vol. 5 (J. Nešetruil and V. Rödl, eds.) 12-28. Berlin: Springer-Verlag, 1990.

[7] P. Erdős, Problems and results in discrete mathematics, Discrete Math. 136 (1994), 53-73.

[8] P. Erdős, A. Hajnal, Ramsey-type theorems, Discrete Appl. Math. 25 (1989), 37-52.

[9] P. Erdős, A. Hajnal, R. Rado, Partition relations for cardinal numbers, Acta Math. Acad. Sci. Hungar. 16 (1965), 93-196.

[10] P. Erdős, R. Rado, Combinatorial theorems on classifications of subsets of a given set, Proc. London Math. Soc. 3 (1952), 417-439.

[11] P. Erdős and G. Szekeres, A combinatorial problem in geometry, Compositio Math. 2 (1935), 463-470.

[12] R.L. Graham, B.L. Rothschild, J.L. Spencer, Ramsey theory, 2nd edition, John Wiley \& Sons (1980).

[13] T. Kövari, V. Sós, and P. Turán, On a problem of K. Zarankiewicz, Colloq Math. 3 (1954), 50-57.

[14] F.P. Ramsey, On a problem of formal logic, Proc. London Math. Soc. Ser. 230 (1930), 264-286.

[15] K. Zarankiewicz, Problem P101, Colloq, Math. 2 (1951), 301. 